using descriptive approaches. Predictors of adverse outcome were examined by logistic regression.

Results 69 (31\%) first pregnancies ended in adverse outcome, including 14 (6\%) with congenital anomalies, 52 (24\%) additional fetal losses, and $3(1 \%)$ additional infant deaths. 41 (19\%) second pregnancies ended in adverse outcome, significantly less than the rate among first pregnancies ( $\mathrm{p}=0.002)$, including $21(10 \%)$ with congenital anomaly, $19(9 \%)$ additional fetal losses, and $1(<1 \%)$ additional infant death. $21(10 \%)$ women experienced an adverse outcome in both pregnancies. Adverse outcome in the first pregnancy was associated with more than double the risk of an adverse outcome in the second pregnancy $[R R=2.3$ (95\% CI 1.3 to 3.9 )]. Compared to those with no history of adverse outcome, women with recurrent adverse outcomes were more likely to be from an ethnic minority background ( $\mathrm{p}=0.01)$.

Conclusion The overall risk of adverse pregnancy outcome is lower in second pregnancies than first, but history of an adverse outcome increases the risk in the second pregnancy.

\section{P1-535 RELATIONSHIP BETWEEN CHARACTERISTICS OF SOCIAL NETWORK, HEALTH-RELATED QUALITY OF LIFE AND MORTALITY PATTERNS IN OLDER AGE. KRAKOW STUDY}

doi:10.1136/jech.2011.142976h.23

B Tobiasz-Adamczyk, ${ }^{*}$ P Brzyski, A Galas, M Brzyska, M Florek. Department of Medical Sociology, Chair of Epidemiology and Preventive Medicine, Jagiellonian University Medical College, Kraków, Poland

Introduction Direct relations between social network and mortality patterns has been well documented, but less is known the relationship between size, density, boundedness of social network, social support, and psycho-social dimensions of quality of life in older age and its direct and indirect effects on all-cause mortality.

Aim The aim of the study was to assess the characteristics of social network in older stage of life in relation to health-related quality of life and its effect on mortality.

Methods The base-line study was performed in simple random sample of 552 Krakow citizen aged $65-85$ yrs old, as a part of Central European Network on Healthy Ageing. Face to face structured interviews were based on Polish validated version of Dutch questionnaire. SF20 test, HADS, GARS, SSL12-I, de Jong-Gierveld Loneliness Scale and Cantril's ladder were used. Data on mortality was ascertained by monitoring city vital records and all-caused mortality was analysed. During 8 years $31.1 \%$ of study population died. Cluster analysis was performed and Cox proportional hazard model was used.

Results Cluster analysis distinguished different clusters for age group -75 yrs and 76 yrs and over. Cox proportional models revealed significantly highest risk of death ( $\mathrm{HR}=2.97,95 \% \mathrm{CI}(1.21$ to 7.28$)$ ) in individuals aged 76 and over who were characterised by seldom contacts with children, neighbours and others, high loneliness, low social support and low general quality of life.

Conclusions Relationship between the properties of social network and health-related quality of life remains significant predictor of mortality in advanced old age.

\section{P1-536 EPIDEMIOLOGY OF PAEDIATRIC BURN INJURIES IN SHIRAZ, IRAN IN 2009}

doi:10.1136/jech.2011.142976h.24

\begin{abstract}
$1,{ }^{2} \mathrm{H}$ Tolide-ie, ${ }^{*} \mathrm{~F}$ Sahraeian, ${ }^{2} \mathrm{Z}$ Afrasiabi, ${ }^{2} \mathrm{~A}$ A Mohammadi, ${ }^{2} \mathrm{M}$ Kherad, ${ }^{2} \mathrm{~S}$ Tolide-ie. ${ }^{1}$ Gonabad University of Medical Sciences, Gonabad, Iran; ${ }^{2}$ Shiraz Burn Research Center, Shiraz University of Medical Sciences, Shiraz, Iran
\end{abstract}

Introduction Burn injuries are very important causes of morbidity and mortality among children. The aim of this study was to determine the burn related injuries in paediatric population in Shiraz, which is one of the largest cities in the Fars province in Iran.

Methods In a cross-sectional study, we investigated 691 burn victims aged 14 years and less in year 2009 admitted to Ghotbeddin hospital, the only burn specialised centre in the South of Iran.

Results These subjects represent 31.5\% (691/2194) of all burn patients treated in that year. The children's mean age was $4 \pm 3.4$ years and their burn per cent was $5.7 \% \pm 10.9 \%$. Their sex ratio $(\mathrm{M} / \mathrm{F})$ was 1.37 and $1-6$ year children were the most frequent group $(58.3 \%)$ among paediatric patients. There were no statistical significant differences in the age $(p=0.67)$ and burn per cent $(p=0.88)$ of two sexes. Only 105 cases $(15 \%)$ were hospitalised and others were treated as out-patients. Five-hundred thirty seven cases $(78 \%)$ were from urban areas and others were rural people. Boiling water $(34 \%)$, hot tea $(20 \%)$ and hot food $(6.8 \%)$ were the three leading causes of paediatric burns. Winter had the highest incidence of paediatric burns (30\%). What's more, living room (45.4\%) and kitchen (31.4\%) were the most common places of burn incidence. The mortality rate in pediatrics was $2 \%$ (14/691).

Conclusion Most of the paediatric burn injuries happened at home by hot liquids in the colder seasons of the year. It is suggested that paediatric burn prevention should be an essential element of parental education.

\section{P1-537 RETINOPATHY OF PREMATURITY AND NEONATAL RISK FACTORS: A RETROSPECTIVE COHORT STUDY}

doi:10.1136/jech.2011.142976h.25

${ }^{1} \mathrm{H}$ Hoseini, ${ }^{1} \mathrm{Z}$ Asadi, ${ }^{2,3} \mathrm{H}$ Tolide-ie, ${ }^{*} \mathrm{~S}$ Kowsarnia. ${ }^{1}$ Department of Ophthalmology, Poostchi Ophthalmic Research Center, Shiraz, Iran; ${ }^{2}$ Gonabad University of Medical Sciences, Gonabad, Iran; ${ }^{3}$ Shiraz Burn Research Center, Shiraz University of Medical Sciences, Shiraz, Iran

Introduction Recent technological advances in neonatology have increased the survival rate of very low birth weight infants, which has led to a correspondingly increased incidence of retinopathy of prematurity (ROP).

Objective To identify neonatal risk factors for severe ROP in premature babies.

Methods A retrospective cohort study was undertaken In Shiraz from January 2006 to April 2010. All premature neonates with gestational age (GA) of $<34$ weeks or birth weight (BW) of $<2000 \mathrm{~g}$ were included in the study.

Results During 5 years of study, 1095 premature infants were analysed. There were $732(66.8 \%)$ infants did not develop ROP, 363 $(33.2 \%)$ infants presented with various degrees of ROP (249 infants without treatment and 114 infants were treated with laser). We compared 2 groups of neonates, who were treated with laser and those who didn't received any treatment. Univariate logistic regression showed that GA $[\mathrm{OR}=0.68(95 \%$ CI 0.62 to $0.75)$ ], $\mathrm{BW}[\mathrm{OR}=0.997$ (95\% CI 0.996 to 0.998$)$ ], length $[\mathrm{OR}=0.84$ (95\% CI 0.80 to 0.89$)$ ], and head circumference (HC) $[\mathrm{OR}=0.73$ (95\% CI 0.66 to 0.80$)$ ] had significant inverse relationships with developing ROP and there is no statistical relationship between sex of neonates and ROP ( $p$ value $=0.054$ ). However, after adjustment in multivariate logistic model, only GA and HC remained in the model as independent risk factors. Adjusted OR for H.C was 0.8 (95\% CI 0.71 to 0.90) and Adjusted ORs for, GA $\leq 28$ weeks, and, 28 weeks $<\mathrm{GA}<32$ weeks, were $4.32(95 \%$ CI 1.76 to 10.57$)$ and 3.5 (95\% CI 1.56 to 7.83$)$ in comparison with , $\mathrm{GA} \geq 32$ weeks.

Conclusion Gestational age and head circumference at birth were the most important risk factors for severe ROP in our study. 